\title{
Traffic-aware Routing Protocol in VANET using Adaptive Autoregressive Crow Search Algorithm
}

\author{
Deepak Rewadkar \\ Department Information Technology \\ Goverment Polytechnic Awasari,India \\ deepakrewadkar27@gmail.com
}

\author{
Dharmpal Doye \\ Department Information Technology \\ Shri Guru Gobind Singhji Institute of Engineering and Technology, \\ Nanded, India
}

\begin{abstract}
The traffic-aware routing in the geographical area is essential in Vehicular Ad Hoc Network (VANETs). VANET is highly significant in the research area for transmitting the sensing information between the vehicular nodes. In VANET, there exist few mobility patterns, which assist to predict the path of the vehicle. Due to the demand in developing the traffic-aware routing protocol in VANET, and effective routing protocol named Adaptive Autoregressive Crow Search optimization Algorithm (Adaptive-ARCSA) is proposed in this work to predict the traffic in road segments. The traffic prediction is achieved by using the Exponential Weighted Moving Average (EWMA) based on the traffic density and vehicle speed. Hence, the optimal path is selected by using the proposed Adaptive- ARCSA by utilizing the objective function. The analysis of the proposed algorithm is made and the performances are evaluated using the metrics, like End to End Delay (EED), average traffic density, distance, and throughput, which acquired with the values of 2,343, 0.034, 2.0896 and 0.1 , respectively.
\end{abstract}

Keywords: Crow Search Algorithm (CSA), traffic prediction, adaptive property, autoregressive model, geographical knowledge.

\section{Introduction}

VANET is an application of the Mobile Ad-Hoc Network (MANET) [8]. VANET is the main core of the Intelligent Transport System (ITS), which is mainly designed to perform the vehicle operation in order to enhance safety in the road. The major advantage of VANET is that it bypasses the infrastructure, as wireless technology is more cheap and pervasive. Due to the device placed or installed at the roadside and vehicles, the vehicular communication warns the drivers regarding the harmful situation and the potential danger [8]. In the vehicular sensing network system, VANET is the suitable means in transmitting the sensing information between the vehicular nodes or between the urban sensing system and the vehicles. VANET contains an On-Board Units (OBU) connected with the roadside units and the vehicles organized in the road. Accordingly, the roadside unit and the OBU have a limited range of communication interfaces. In VANET, the information is broadcasted between the vehicles (vehicle to vehicle communication or V2V) till the receiver received the message within the transmission range, and after that, they are broadcasted to the roadside unit [5]. VANET offers more sophisticated services, like weather conditions, internet service, and traffic conditions to enhance the safety of the road. Vehicles are self-organized and autonomous in nature to exchange information among the vehicles. VANET has unique features, which includes topology changes and vehicle speed. Forwarding the data and routing the packets in VANET is a quite challenging task [8] [18] [19].

VANET focused on acute technology in the smart system. It is the most significant research field evolving in the field of the network to attain safe driving, and traffic optimization [7]. Most of the effective approaches are introduced to manage and maintain the vehicle to automatically reduce the accidents and traffic jams [7]. In VANET, there exist few mobility patterns [20] [21] [22] due to the social features of the humans, which help to predict the path of the vehicle. In VANET, routing algorithms are developed, which functions based on the vehicle path predictability. Trajectory-Based Data forwarding scheme (TBD) forwards the data for the roadside report in the light traffic VANET. In the TBD scheme, each individual vehicle 
calculates the delivery delay based on the path of the vehicle to predict the next-hop vehicle [5]. Traditional routing protocol faces a lot of problems while dealing with the vehicular network with high mobility speed [8]. The requirements of the vehicular network are entirely different than the mobile ad hoc network. The energy consumption and the memory storage in VANET are not constant, as the storage space and the battery power are unlimited [28]. Due to high mobility, the vehicles are not randomly moving, but it follows the mobility pattern of the general networks [8] [17]. In VANET, the information can only be forwarded through the road, hence the geography topology structure have a strong collision in the performance of the packet transmission [23] [24] [25]. Based on the knowledge of the geographical structure, the greedy perimeter routing protocol is introduced to forward the messages [5] [26] [27].

The primary intention of this work is to design and develop an effective traffic-aware routing protocol by using the proposed Adaptive-ARCSA in the geographical area. Initially, the VANET is passed into the traffic prediction, where the EWMA approach is utilized to achieve traffic prediction in the road segments. The predicted traffic flow is further sent to the optimal path selection, where the proposed Adaptive-ARCSA is used to progress the path selection mechanism, by incorporating the adaptive features into the autoregressive model. This paper is organized as follows: Section 2 describes the Literature survey of the paper and section 3 describes the proposed Adaptive Autoregressive Crow Search Algorithm for traffic-aware routing. Section 4 states the results and discussion of the paper and section 5 defines the conclusion of the paper.

\section{Literature Survey}

Various literature surveys are reviewed in this section. Liu C et al. [1] modeled a stable directional forward routing algorithm to achieve reliable and secure routing framework in the Vehicular Ad-hoc network. The node present in the specified range participates into the routing process to minimize the frequency of requests. It attained better performance but failed to consider the delay. Zhu Y et al. [2] developed a roadbased routing algorithm to compute the routing path. It effectively works well under various network conditions. However, it attained minimal delivery delay but does not explicitly transmit the packets. Karimi R. and Shokrollahi S [3] developed a predictive geographic routing protocol to enhance the connectivity of the nodes. Here, the vehicle assigns the weight to the neighboring vehicle based on the angle and direction of the vehicle. However, it attained better delivery ration, but the performance was degraded. Khan S et al. [4] developed Traffic-aware Segment Based Routing (TASR) protocol to perform the routing framework in VANET. It computes the connectivity degree for different segments and uses the forwarding model to transmit the data packets from the source to the destination based on the geographical information. However, it attained a better delivery ratio, but the network overhead was not eliminated.

\subsection{Challenges}

- Attaining the feasibility in the multi-hop communication results a challenging task in VANET, as a number of vehicles participate in the network [1].

- The topology structure of VANET is highly dynamic and changes over times. However, it frequently blocks the communication in the vehicular network is a major challenge associated with the network [4].

- The routing path between the vehicular nodes to broadcast the data packets with more reliable is a challenging task associated with the VANET network [10].

\section{Proposed Adaptive Autoregressive Crow Search Algorithm for Traffic-Aware Routing}

VANETS used in the traffic aware routing mechanism is initially sent to the traffic prediction, where the EWMA is used to predict the traffic in the network route by using the average traffic density and the average vehicle speed. The optimal path selection is performed by using the proposed algorithm, which generates k-paths in the network route. Hence, the optimal path is selected from the generated k-paths by using the objective function. The objective function is mainly utilized to reduce the end to end delay, maximize the link lifetime, minimize the distance, and reduce the packet delay. The proposed algorithm uses 
three adaptive factors, like distance, fitness, and iterative to ensure efficient traffic-aware routing in VANET.

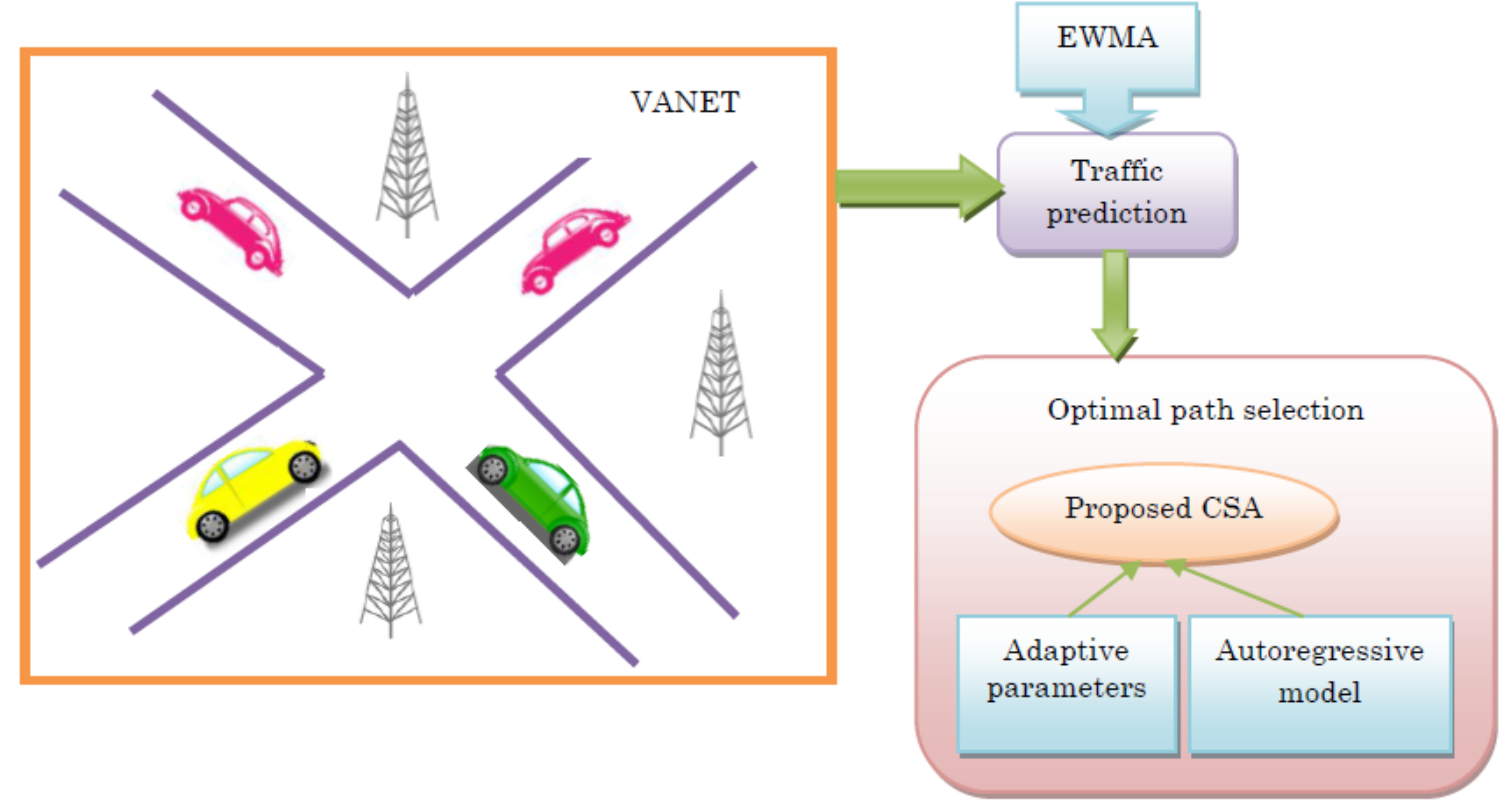

Fig. 1. Proposed Adaptive Autoregressive Crow Search Algorithm for traffic-aware routing

\section{a) Traffic prediction using EWMA}

The path selection is performed by using the EWMA, which determines the traffic density and the traffic path based on the previous data. The logic is expressed as,

$$
\mathrm{c}(\mathrm{f}+1)=\lambda \mathrm{z}(\mathrm{f}+1)+(1-\lambda) \mathrm{c}(\mathrm{f}) ;(1 \leq \mathrm{f} \leq \mathrm{r})
$$

where, $r$ determines the number of observations, $\lambda$ is a constant which varies from 0 to 1 . The observation time of $(f+1)$ is given as $z(f+1)$, and the exponential weight at the time $f$ and $(f+1)$ is denoted as $\mathrm{c}(\mathrm{f})$ and $\mathrm{c}(\mathrm{f}+1)$.

\section{b) Adaptive Autoregressive Crow search algorithm}

Crow Search Algorithm (CSA) [16] is a meta-heuristic optimization algorithm, where the crow stores their excess food in the hidden location and retrieves it back when it is required. CSA have different features, like constraints, decision variables, and objective functions are utilized to solve the optimization problems. The optimization algorithm is mainly used to compute the best solution from the available solutions. CSA is more effective in finding the optimal solution, as the crows are broadly dispersed species of birds. Crows score high performance in the intelligence tests and are considered as the intelligent animal in the worldwide. Crows use the tools, memorize the faces, hide and rescue their food sources, and communicate with others in a sophisticated manner. Based on the behavior of the crow flock, the crow hides their food in some hidden places in the environment and retrieves back the food only when it is required. Crows are considered greedy birds, as it follows the path of other birds to get the optimal food source. In the optimization observance, the crows are referred to as the searchers, the environment is defined as the search space, and the environmental position corresponds to the feasible solution. The fitness function of the crow is computed by using the quality of the food source, and the best source is considered as the optimal solution for the optimization problem.

Crows have the largest brain size, which is related to the body size of the crow, as it is the most intelligent birds among the animals. Based on the brain to body ratio, it is clearly specified that the brain of the crow is a little lower than the brain of a human. Most evidence is demonstrated that the crows are most clever in the mirror test and have better tool-making capability. When a un-friend one meets, the crows remember their faces and warn them in the environment. The crows observe the location of food of other crows; they will steal the food when the owner crow leaves from the position. The crows live in the flock form, remember their food location, and protect their own food source by hiding the food location. Let us assume that, $\mathrm{n}$ dimensional environment contains a number of crows. Here, the flock size or the number of 
crows is denoted as $A$ and the position of the crow id indicated as $\mathrm{m}$ with the time itr in the search space is represented as, $\mathrm{E}^{\mathrm{m}, \mathrm{itr}}(\mathrm{m}=1,2, \ldots ., \mathrm{A}) ; \mathrm{itr}=1,2, \ldots ., \mathrm{itr}_{\max }$, where $\mathrm{E}^{\mathrm{m}, \mathrm{itr}}=\left[\mathrm{E}_{1}^{\mathrm{m}, \mathrm{itr}}, \mathrm{E}_{2}^{\mathrm{m}, \mathrm{itr}}, \ldots \ldots . ., \mathrm{E}_{\mathrm{n}}^{\mathrm{m}, \mathrm{itr}}\right]$ and itr $_{\max }$ is the maximum iterations.

Each and every crow has its own memory location and so the hidden place of the food is memorized. Crows move in the search space and environment to find the optimal food source.

Let us consider that at iteration itr the crow $\mathrm{p}$ visit the hidden location $\mathrm{a}^{\mathrm{p}, \mathrm{itr}}$ and also the crow $m$ follows the crow $\mathrm{p}$ to find the food source of crow $\mathrm{p}$. In this scenario, two different states occur in the CSA algorithm is elaborated as below:

State 1: The crow $p$ never knows that the crow $m$ is following crow $p$, hence the new location of crow $m$ is represented as,

$$
\mathrm{E}^{\mathrm{m}, \mathrm{itr}+1}=\mathrm{E}^{\mathrm{m}, \mathrm{itr}}+\mathrm{b}_{\mathrm{m}} \times \mathrm{g}^{\mathrm{m}, \mathrm{itr}} \times\left(\mathrm{a}^{\mathrm{p}, \mathrm{itr}}-\mathrm{E}^{\mathrm{m}, \mathrm{itr}}\right)
$$

where, $b_{m}$ denotes the random number which lies in the range between 0 and 1 , and $g$ represents the flight length of the crow $m$ at iteration itr .

State 2: The crow $\mathrm{p}$ knows that the crow $\mathrm{m}$ is following it. Hence, the crow $\mathrm{p}$ tries to protect its food to make the crow $j$ fool by moving to another location. Hence, the total state is expressed as,

$$
\mathrm{E}^{\mathrm{m}, \text { itr }+1}=\left\{\begin{array}{lr}
\mathrm{E}^{\mathrm{m}, \text { itr }}+\mathrm{b}_{\mathrm{m}} \times \mathrm{g}^{, \text {itr }} \times\left(\mathrm{a}^{\mathrm{p}, \text { itr }}-\mathrm{E}^{\mathrm{m}, \text { itr }}\right) & \mathrm{b}_{\mathrm{p}} \geq \mathrm{M}^{\mathrm{p}, \text { itr }} \\
\text { a random position } & \text { otherwise }
\end{array}\right.
$$

where, $b_{p}$ denotes the random number varies from 0 to 1 , and $\mathrm{M}^{\mathrm{m} \text {,itr }}$ represents the awareness probability of the crow $\mathrm{p}$ at iteration itr. The awareness probability parameter is used to control the diversification and the intensification of the crow, when the value of $M$ decreases, the CSA searches their food in the local region, and the value of intensification increases. When the awareness probability increases, the searching probability decreases, and the CSA explore their scale in the search space.

The autoregressive model is represented as,

$$
\mathrm{E}(\mathrm{f})=\mu_{0}+\sum_{\mathrm{f}=1}^{1} \mu_{\mathrm{f}} \mathrm{E}(\mathrm{f}+1)=\sum_{\mathrm{i}=1}^{\mathrm{n}} \mu_{\mathrm{i}} \mathrm{d}\left(\mathrm{E}_{\mathrm{f}} \text { i }\right)
$$

where, $\mu_{0}$ denotes the unknown parameter, $l$ and $n$ represents the constants. $E(f-1)$ indicates the agent location, the dimension of the parameter $\mu=1+\mathrm{n}=1$, and $d\left(E_{f-i}\right)$ denotes the fitness.

The procedures involved in the CSA optimization are described as below:

\section{Step 1: Problem initialization}

Initially, the decision variables, optimization problem, and the constraints are explained. Thereafter, the number of iterations itr, flight length $\mathrm{g}$, awareness probability M, and flock size A are defined.

\section{Step 2: Memory and position initialization of the crow}

In the $n$ dimensional search space A number of crows is randomly placed, where each crow defines the best solution using $n$ decision variable.

$$
\text { crows }=\left[\begin{array}{ccccc}
\mathrm{E}_{1}^{1} & \mathrm{E}_{2}^{1} & \ldots \ldots . & \mathrm{E}_{\mathrm{n}}^{1} \\
\mathrm{E}_{1}^{2} & \mathrm{E}_{2}^{2} & \ldots \ldots . & \mathrm{E}_{\mathrm{n}}^{2} \\
\cdot & \cdot & \cdot & & \cdot \\
\cdot & \cdot & \cdot & & \cdot \\
\cdot & \cdot & \cdot & & \cdot \\
\mathrm{E}_{1}^{\mathrm{A}} & \mathrm{E}_{2}^{\mathrm{A}} & \ldots \ldots . & \mathrm{E}_{\mathrm{n}}^{\mathrm{A}}
\end{array}\right]
$$

The crows hide their location of food at the initial position and the memory is also initialized at the initial iteration.

\section{Step 3: Fitness evaluation}

The decision variable is inserted into the objective function to compute the quality of the position for each crow. 


\section{Step 4: New position generation}

When a crow wants to find a new position, then the cow randomly follows the position of other crow discover the new position. The newly generated position of the crow $\mathrm{m}$ is defined in the Eq. (3)

\section{Step 5: Verify the feasibility of the new position}

For each row, the feasibility of the new location is checked. If the new location of the crow is feasible, then the crow updates their position otherwise, the crow does not shift to the new location it still remains in the old position.

\section{Step 6: Fitness evaluation for a new position}

For each crow, the fitness function for the newly generated position is calculated.

\section{Step 7: Memory update}

The memory of the crow is updated using the below equation,

$$
a^{m, i t r+1}= \begin{cases}E^{m, i t r+1} & d\left(E^{m, i t r+1}\right) \text { isbetterth and }\left(a^{m, i t r}\right) \\ a^{m, i t r} & \text { otherwise }\end{cases}
$$

where, $\mathrm{d}($.$) represents the objective function.$

It is clearly shown that, if the fitness function of the newly generated position is better than the fitness function of the memory location, then the crow updates their memory with the newly generated position.

\section{Step 8: Termination}

The above steps are repeated until the maximum iteration itr $_{\max }$ is attained. Once the termination criteria are reached, the best memory position with respect to the objective function is declared as the optimal solution for the optimization problem.

\section{Results and Discussion}

\subsection{Experimental Setup}

The implementation of the proposed algorithm is carried out in MATLAB tool, which operates with Intel i-3 processor, 2GB RAM, and Windows 8 OS with the simulation analysis of 50 vehicles with respect to the simulation time as 50 seconds.

\subsection{Evaluation Metrics}

The metrics used to analyze the performance of the proposed approach is explained as below:

EED is defined as the time taken to reach the destination node from the source node, and throughput defines the packets that are transmitted to the destination at the simulation time. The traffic density indicates the total number of vehicles present in the road at a certain time, and distance refers to the length of the road segment.

\subsection{Comparative Methods}

The performance of the proposed approach is analyzed and is compared with the existing methods, namely Stable CDS Based Routing Protocol (SCRP) [14], Glow Worm Swarm Optimization (GWSO) [15], Fractional Glowworm Swarm Optimization (FGWSO), Whale Optimization Algorithm (WOA) [13], Autoregressive Based WO (ARWO), Adaptive ARWO, respectively.

\subsection{Comparative Analysis}

The analysis made using 50 vehicles for the metrics, EED, distance, throughput, and the traffic density is explained in this section. Fig. 2 a) shows the analysis of throughput with respect to the time. The throughput attained by the existing methods, like SCRP, GWSO, FGWSO, WOA, ARWO, Adaptive-ARWO is 0.42, 0.52, $0.55,0.45,0.59$, and 0.677 with respect to the time as $10 \mathrm{sec}$. The proposed Adaptive ARCSA attained better 
throughput than the existing methods with a value of 0.7 for $10 \mathrm{sec}$. Fig. $2 \mathrm{~b}$ ) shows the analysis of average traffic density with respect to the time. For $30 \mathrm{sec}$, the average traffic density attained by the existing methods, like SCRP, GWSO, FGWSO, WOA, ARWO, Adaptive-ARWO is 0.025, 0.016,0.016, $\quad 0.014, \quad 0.014$, and 0.014, respectively, while the proposed Adaptive ARCSA obtained better traffic density as 0.011, respectively. Fig. 2 c) shows the analysis of distance with respect to the vehicle. For 30 vehicle, the distance attained by the existing methods, namely SCRP, GWSO, FGWSO, WOA, ARWO, Adaptive-ARWO 4.1974, $10.711,4.097,4.88,1.11$, and 1.1101, whereas the proposed Adaptive-ARWO obtained better distance with the value of 4.11, respectively. Fig. 2 d) shows the analysis of EED with respect to the vehicle. For 50 vehicles, the EED computed by the existing methods, like SCRP, GWSO, FGWSO, WOA, ARWO, AdaptiveARWO is 2.9412, 14.706, 2.9412, 6.275, 2.9412, and 2.9382, while the proposed Adaptive-ARWO attained the EED as 2.3482 , respectively.

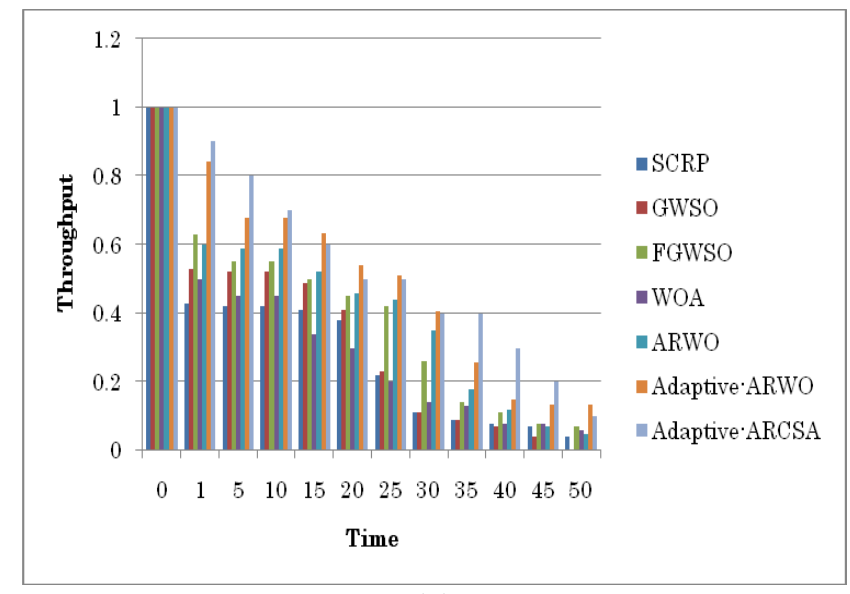

(a)

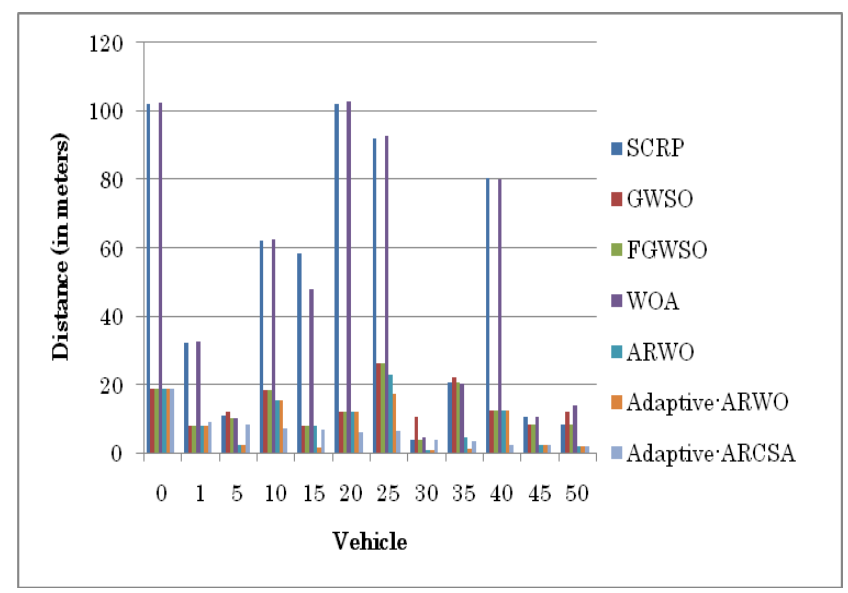

(c)

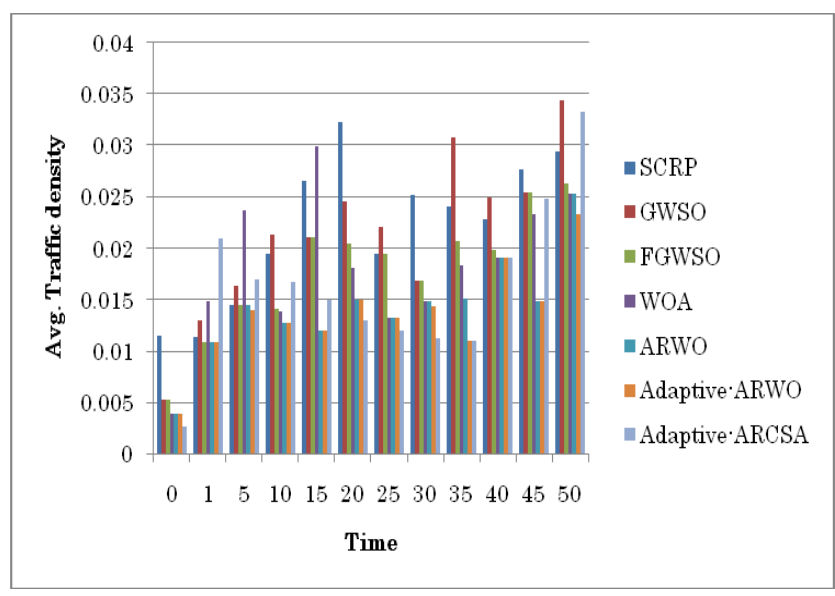

(b)

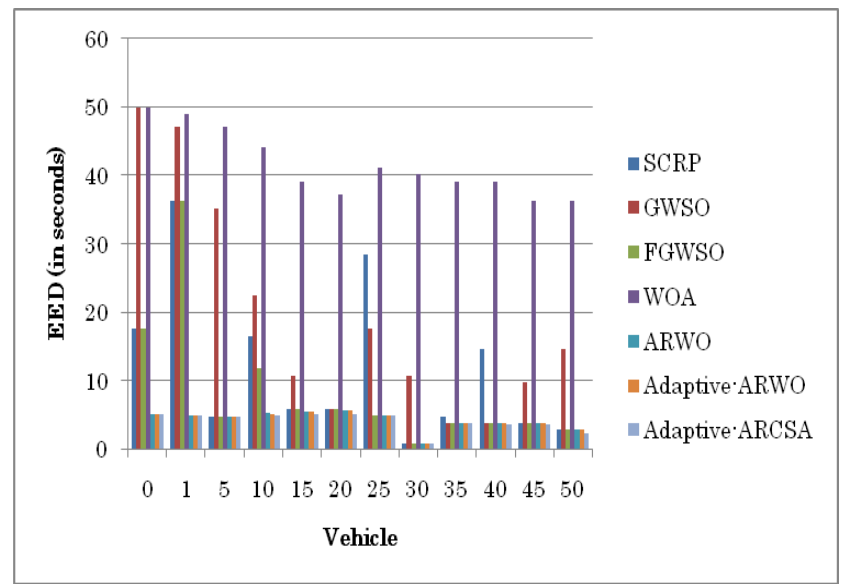

(d)

Fig. 2. Comparative analysis using 50 vehicles, a) Throughput, b) Traffic density, c) distance, d) EED

\section{Conclusion}

VANETs are represented as one of the enabling technologies to evade road accidents by permitting the vehicles to distribute the traffic-related information between them. In addition, the VANETs is exploited for traffic management and infotainment applications. Here, the traffic-aware routing protocol named Adaptive Autoregressive Crow Search Optimization Algorithm is proposed in this work. The traffic prediction is progressed by using the Exponential Weighted Moving Average based on the traffic density and vehicle speed. Moreover, the optimal path is selected by using the proposed Adaptive Autoregressive Crow Search optimization Algorithm by utilizing the objective function. The proposed algorithm attained better performance as 2,343, 0.034, 2.0896 and 0.1 for End to End delay, average traffic density, distance, and throughput, respectively. 
Traffic-aware Routing Protocol in VANET using Adaptive Autoregressive Crow Search Algorithm

\section{Compliance with Ethical Standards}

Conflicts of interest: Authors declared that they have no conflict of interest.

Human participants: The conducted research follows the ethical standards and the authors ensured that they have not conducted any studies with human participants or animals.

\section{References}

[1] Liu C., Shu Y., Yang O., Xia Z. and Xia R, "SDR: a stable direction-based routing for vehicular ad hoc networks", Wireless Personal Communications, vol. 73, no. 3, pp.1289-1308, 2013.

[2] Zhu Y., Qiu Y., Wu Y., Gao M., Li B. and Hu Y., "On adaptive routing in urban vehicular networks", Wireless networks, vol. 19, no. 8, pp.1995-2004, 2013.

[3] Karimi R. and Shokrollahi S., "PGRP: Predictive geographic routing protocol for VANETs", Computer Networks, vol. 141, pp.67-81, 2018.

[4] Khan S., Alam M., Fränzle M., Müllner N. and Chen Y., "A Traffic Aware Segment-based Routing protocol for VANETs in urban scenarios", Computers \& Electrical Engineering, vol. 68, pp.447-462, 2018.

[5] Yu D.I.N.G., LIU Y.Z., GONG X.Y. and WANG W.D., "Road traffic and geography topology based opportunistic routing for VANETs", The Journal of China Universities of Posts and Telecommunications, vol. 21, no. 4, pp.32-39, 2014.

[6] Taleb T., Sakhaee E., Jamalipour A., Hashimoto K., Kato N. and Nemoto Y., "A stable routing protocol to support ITS services in VANET networks", IEEE Transactions on Vehicular technology, vol. 56, no. 6, pp.3337-3347, 2007.

[7] Das D. and Misra R., "Improvised dynamic network connectivity model for Vehicular Ad-Hoc Networks (VANETs)", Journal of Network and Computer Applications, vol. 122, pp.107-114, 2018.

[8] Boussoufa-Lahlah S., Semchedine F. and Bouallouche-Medjkoune L., "Geographic routing protocols for Vehicular Ad hoc NETworks (VANETs): A survey", Vehicular Communications, vol. 11, pp.20-31, 2018.

[9] Rehman O. and Ould-Khaoua M., "A hybrid relay node selection scheme for message dissemination in VANETs", Future Generation Computer Systems, vol. 93, pp.1-17, 2019.

[10] Kumari N.D. and Shylaja B.S., "AMGRP: AHP-based multimetric geographical routing protocol for urban environment of VANETs", Journal of King Saud University-Computer and Information Sciences, 2017.

[11] Song C., Wu J., Liu M. and Zheng H., "Efficient routing through discretization of overlapped road segments in VANETs", Journal of parallel and distributed computing, vol. 102, pp.57-70, 2017.

[12] Chai H., Zhang H.M., Ghosal D. and Chuah C.N., "Dynamic traffic routing in a network with adaptive signal control”, Transportation Research Part C: Emerging Technologies, vol. 85, pp.64-85, 2017.

[13] S. Mirjalili, A. Lewis, "The Whale Optimization Algorithm", Advances in Engineering Software, vol. 95, pp. 51-67, 2016.

[14] M.A. Togou, AbdelhakimHafid, and LyesKhoukhi, "SCRP: Stable CDS-Based Routing Protocol for Urban Vehicular Ad Hoc Networks", IEEE Transactions on Intelligent Transportation Systems, vol. 17, no. 5, pp. 1298-1307, 2015.

[15] B. Wu, C. Qian, W. Ni, S. Fan, "The improvement of glow worm swarm optimization for continuous optimization problems", Expert Systems with Applications, vol. 39, no. 7, pp. 6335-6342, 2012.

[16] C. Qu and Y. Fu, "Crow Search Algorithm Based on Neighborhood Search of Non-Inferior Solution Set," in IEEE Access, vol. 7, pp. 52871-52895, 2019.

[17] Vijayakumar Polepally, K Shahu Chatrapati,"DEGSA-VMM: Dragonfly-based exponential gravitational search algorithm to VMM strategy for load balancing in cloud computing";Kybernetes, vol.67, no.6;pp.1138-1157;2018.

[18] D. Menaga and Dr.S. Revathi,"Privacy Preserving using Bio Inspired Algorithms for Data Sanitization",International Conference on Electrical, Electronics, Computers, Communication, Mechanical and Computing (EECCMC); pp. 201-206, 2018.

[19] RV Ravi, K Subramaniam, "Image Compression and Encryption using Optimized Wavelet Filter Bank and Chaotic Algorithm",International Journal of Applied Engineering Research, vol. 12, no. (21); pp. 10595-10610, 2017.

[20] S Chander, P Vijaya, P Dhyani,"Multi kernel and dynamic fractional lion optimization algorithm for data clustering", Alexandria engineering journal, vol. 57, no (1), pp.267-276, 2016.

[21] M Mukhedkar, P Powar, P Gaikwad, "Secure non real time image encryption algorithm development using cryptography \& Steganography",2015 Annual IEEE India Conference (INDICON), pp. 1-6, 2015.

[22] RM Chintalapalli, VR Ananthula,"M-LionWhale: multi-objective optimisation model for secure routing in mobile adhoc network",IET Communications,vol. 12, no.(12), pp.1406-1415,2018.

[23] MNKMSS Dr. N. Krishnamoorthy,"Performance Evaluation of Optimization Algorithm Using Scheduling Concept in Grid Environment", The IIOAB Journal 7 (9), pp. 315-323, 2016.

[24] SB Vinay Kumar, PV Rao, Manoj Kumar Singh,"Multi-culture diversity based self adaptive particle swarm optimization for optimal floorplanning",Multiagent and Grid Systems, vol14, no.1, pp.31-65, 2018. 
[25] Archana H. Sable Haricharan Dhirbasi,Dr. Bondar Kirankumar Laxmanrao,"Application of Integral Transform to Recognition of Plastic Surgery Faces and the Surgery Types: an Approach with Volume based Scale Invariant Features and SVM",vol.6, no.3, pp.1061-1072, 2018.

[26] R Gupta Roy, D Baidya,"Speed Control of DC Motor Using Fuzzy-Based Intelligent Model Reference Adaptive Control Scheme",Advances in Communication, Devices and Networking, Lecture Notes in Electrical Engineering book series, Springer, vol. 462, pp.729-735, 2018.

[27] G Singh, VK Jain, A Singh, "Adaptive network architecture and firefly algorithm for biogas heating model aided by photovoltaic thermal greenhouse system",Energy \& Environment, vol. 29 (7), pp.1073-1097, 2018.

[28] A Shankar, J Natarajan,"Base Station Positioning in Wireless Sensor Network to aid Cluster Head Selection Process", International Journal of Intelligent Engineering and Systems", vol. 10, no.(2), pp.173-182, 2017. 